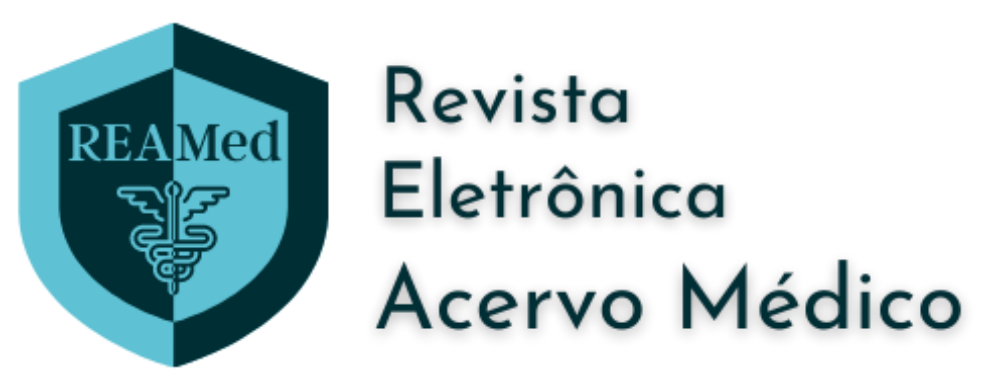

\title{
Síndrome Inflamatória Multissistêmica Pediátrica e Doença de Kawazaki: as diferenças e manifestações clínicas na Pediatria
}

\author{
Pediatric Multisystem Inflammatory Syndrome and Kawazaki Disease: the differences and \\ clinical manifestations in Pediatrics
}

Síndrome Inflamatorio Multisistémico Pediátrico y Enfermedad de Kawazaki: diferencias y manifestaciones clínicas en Pediatría

Mariana Silva dos Santos ${ }^{1 *}$, Suellen Sthefany Mota Tiago Andrighetto'.

\section{RESUMO}

Objetivo: Descrever os principais fatores relacionados à Síndrome Inflamatória Multissistêmica Pediátrica Associada ao Covid-19 (SIM-P) e suas complicações no cenário atual, bem como as suas principais diferenças com a Doença de Kawazaki (DK). Revisão bibliográfica: A pesquisa foi realizada de forma exploratória no contexto dos acontecimentos atuais, e nas opiniões científicas encontradas na atualidade sobre as principais manifestações clínicas da SIM-P que são: hipertermia, sintomas abdominais, manifestações cutâneas, linf adenopatia generalizada, alterações cardíacas e nervosas e as manifestações da DK. Considerações finais: Apesar da evidência científica limitada disponível, acredita-se que haja uma inflamação na infância que pode estar associada à infecção por SARS-CoV-2, semelhante à da doença de Kawasaki. Exame clínico completo, incluindo anamnese, exame físico, vínculo epidemiológico e casos suspeitos ou confirmados de Covid-19 nas últimas 2 a 4 semanas, além da solicitação de exames complementares para avaliação do processo inflamatório e envolvimento do órgão, é fundamental para suspeitas diagnóstico e tratamento.

Palavras-chave: COVID-19, Unidades de terapia intensiva pediátrica, Síndrome de linfonodos mucocutâneos, Síndromes periódicas associadas à criopirina.

\section{ABSTRACT}

Objective: To describe the main factors related to the Pediatric Multisystem Inflammatory Syndrome Associated with Covid-19 (SIM-P) and its complications in the current scenario, as well as its main differences with Kawazaki Disease (KD). Bibliographic review: The research was carried out in an exploratory way in the context of current events, and in the scientific opinions found today about the main clinical manifestations of SIM-P, which are: hyperthermia, abdominal symptoms, cutaneous manifestations, generalized lymphadenopathy, cardiac and nervous alterations and the manifestations from DK. Final considerations: Despite the limited scientific evidence available, it is believed that there is childhood inflammation that may be associated with SARS-CoV-2 infection, similar to Kawasaki disease. Complete clinical examination, including anamnesis, physical examination, epidemiological link and suspected or confirmed cases of Covid-19 in the last 2 to 4 weeks, in addition to the request of complementary tests to evaluate the inflammatory process and organ involvement, is essential for suspected diagnosis and treatment.

Key words: COVID-19, Intensive care units pediatric, Mucocutaneous lymph node syndrome, Cryopyrinassociated periodic syndromes.

${ }^{1}$ Universidade Federal do Mato Grosso do Sul (UFMS), Campo Grande - MS. *E-mail: mariana.ipg@hotmail.com SUBMETIDO EM: 1/2022 


\section{RESUMEN}

Objetivo: Describir los principales factores relacionados con el Síndrome Inflamatorio Multisistémico Pediátrico Asociado a Covid-19 (SIM-P) y sus complicaciones en el escenario actual, así como sus principales diferencias con la Enfermedad de Kawazaki (EK). Revisión bibliográfica: La investigación se realizó de manera exploratoria en el contexto de los acontecimientos actuales, y en las opiniones científicas encontradas hoy sobre las principales manifestaciones clínicas de SIM-P, que son: hipertermia, síntomas abdominales, manifestaciones cutáneas, adenopatías generalizadas, alteraciones cardíacas y nerviosas y las manifestaciones de DK. Consideraciones finales: A pesar de la evidencia científica limitada disponible, se cree que existe una inflamación infantil que puede estar asociada con la infección por SARS-CoV-2, similar a la enfermedad de Kawasaki. El examen clínico completo, incluyendo anamnesis, examen físico, nexo epidemiológico y casos sospechosos o confirmados de Covid -19 en las últimas 2 a 4 semanas, además de la solicitud de exámenes complementarios para evaluar el proceso inflamatorio y compromiso de órganos, es fundamental para los casos sospechosos diagnóstico y tratamiento.

Palabras clave: COVID-19, Unidades de cuidado intensivo pediátrico, Síndrome mucocutáneo linfonodular, Síndromes periódicos asociados a criopirina.

\section{INTRODUÇÃO}

Em 2019, teve início uma série de casos na cidade de Wuhan, província de Hubei, na China, de uma nova doença que atinge principalmente o aparelho respiratório com complicações graves. Constatou-se que a transmissão dessa doença é causada pelo contato com uma pessoa infectada, através de partículas contaminadas quando uma pessoa tosse, espirra ou por secreção nasal (CHEN N, et al., 2020).

Devido a esses fatores, a doença se espalhou rapidamente pelo país, que, com a globalização mundial e o encurtamento do tempo e da distância, provocados pela industrialização (carros, trens e aviões), foi rapidamente espalhada para o mundo todo, provocando uma pandemia no cenário mundial e uma grave crise de saúde pública. As pessoas foram acometidas com pneumonias com elevado número de óbitos na população em um curto espaço de tempo, gerando medo e medidas extremas por parte dos governos, como o lockdown, que é confinamento das pessoas em suas residências, e a proibição dos serviços não essenciais, o que gerou uma grave crise econômica e social (SWANN OV, et al., 2020).

Com o número de casos aumentando de maneira avassaladora dia a dia, houve um esforço conjunto mundial para se descobrir mais sobre a doença, a prevenção, a transmissão e o tratamento. De imediato buscou-se produzir uma vacina que coloca-se um ponto final ao número assustador de óbitos entre os infectados (VIEIRA RSR, et al., 2020).

A transmissão denominada Covid-19, ou Severe Acute Respiratory Syndrome Coronavirus 2 (SARS-CoV2), af eta principalmente pacientes adultos, o que levou a princípio os pesquisadores a acreditarem que as crianças eram pouco af etadas e/ou assintomáticas, com baixo risco de gravidade. No entanto, com o avanço da doença, que se tornou uma pandemia, os registros de pacientes pediátricos afetados por uma grave resposta inflamatória multissistêmica, aproximadamente quatro semanas após a infecção aguda por SARS CoV-2, despertaram a preocupação de vários pesquisadores e profissionais da área da saúde à fisiopatologia associada a esta complicação (CATTALINI M, et al., 2021).

Com isso, a pesquisa sobre a doença no setor pediátrico tornou-se essencial. Os primeiros registros da síndrome ocorreram na Itália, Reino Unido e Estados Unidos da América. No Brasil, de 10 de abril de 2020 a 13 de fevereiro de 2021, segundo boletim epidemiológico do Ministério da Saúde (MS), foram confirmados 736 casos dessa síndrome e, destes, 46 evoluíram para óbito, com 6,3\% de letalidade (HAN Q, et al., 2020).

Entre o perfil dos pacientes, houve predomínio do sexo masculino $(56,3 \%)$ e crianças de 0 a 4 anos $(41,4 \%)$. Dentre os casos notificados, $30 \%$ das crianças apresentavam alguma comorbidade e mais de $61 \%$ dos pacientes necessitaram de internação em Unidade de Terapia Intensiva (UTI). Os pacientes apresentavam sintomas semelhantes à Doença de Kawasaki (DK), síndrome do choque tóxico e síndrome hemofagocítica, como presença de alterações cutâneas e comprometimento de órgãos vitais após infecção 
por SARS-CoV-2. Assim, o grupo de manifestações clínicas que afetam vários órgãos após a infecção por SARS-COV-2 foi denominado Síndrome Inflamatória Multissistêmica em Crianças (SIM-P) (ALONSO IAF, 2021).

Com isso, o objetivo deste estudo foi descrever os principais fatores relacionados à SIM-P e suas complicações no cenário atual, bem como as suas principais diferenças com a DK, por intermédio de uma revisão bibliográfica narrativa.

\section{REVISÃo BIBLIOGRÁFICA}

A DK af eta principalmente crianças com menos de cinco anos. É uma vasculite inflamatória aguda que af eta vasos pequenos e médios, de etiologia desconhecida. É uma vasculite aguda e multissistêmica que af eta os vasos de médio porte. É muito comum em crianças, principalmente em menores de cinco anos, mas há relatos em livros de casos para adultos. A DK pode causar vasculite em vários órgãos, como pulmão, intestino, vesícula biliar, sistema nervoso central, entre outros, mas o envolvimento cardíaco é mais importante, com a formação de aneurismas coronários. O diagnóstico é clínico e o tratamento medicamentoso, assim que iniciado, leva à melhora clínica e reduz o risco de sequelas cardíacas (SARZAEIM M e REZAEI N, 2020).

De acordo com um procedimento estabelecido pela American Heart Association (AHA) e pela European League Against Rheumatism/Pediatric Rheumatology European Society (EULAR/PReS), o diagnóstico daDK é, na verdade, clínico e inclui: febre que persiste por, pelo menos, cinco dias, acompanhados por quatro ou mais destes sinais clínicos: exantema (maculopapular difuso é o mais comum); alteração de extremidades (eritema, edema, dor ou descamação); conjuntivite bilateral não exsudativa; alteração em cavidade oral (eritema, fissura, descamação, língua em framboesa, sangramento de lábios) ou linfadenopatia cervical (aumento de mais de um linfonodo maior ou igual a 1,5 cm) (KABEERDOSS J, et al., 2020).

Dentre os achados laboratoriais, destaca-se o aumento dos marcadores inflamatórios, taxa de Sedimentação Eritrocitária (VHS) e Proteína C Reativa (PCR), hiponatremia, leucocitose com neutrofilia acompanhada de anemia grave. Os pacientes que não atendem a todos os sinais clínicos são classificados como DK anormal ou incompleta. A DK incompleta deve ser suspeitada na presença de febre inexplicada (5 dias ou mais) associada a dois ou mais métodos de diagnóstico clínico estabelecidos (WANG Y, et al., 2020).

Na Síndrome Inflamatória Multissistêmica, os sintomas são febre, diarreia e dor abdominal, erupção cutânea, vômitos, cefaleia, sintomas respiratórios, em uma parcela significativa dos pacientes pediátricos, ataques de pânico. Modificação laboratorial indica linfopenia, neutrofilia, aumento de ferritina, dímero-D, proteína $C$ ativa e troponina, além de anemia e trombocitopenia (HOANG A, et al., 2020).

De acordo com Alonso IAF (2021), as manif estações e tratamento, na maioria casos, é: choque que requer suporte com inotrópicos; miocardite e insuficiência cardíaca, frequentemente exigindo suporte respiratório e aminas vasopressoras. O uso de swab nasal para detecção da infecção pelo SARS-CoV-2 não é indicado nesta, pois a SIM-P geralmente se manifesta em média, 14 dias da infecção. No entanto, um grande número de pessoas na faixa etária de crianças com SIM-P testado estava presente com Covid-19, indicando uma infecção recente.

A manifestação de Covid-19 na infância é muito baixa. Em uma série de estudos chineses envolvendo crianças com menos de 19 anos foram encontrados apenas $2 \%$ de casos. Em um estudo de grupo envolvendo crianças (idades $0-18$ ) na Itália, 1,2\% foram af etados. Na Coreia, 4,8\% das pessoas com menos de 19 anos de idade foram testadas e diagnosticadas (FELDSTEIN LR, et al., 2020).

O registro existente a Covid-19 é limitada em estudos pediátricos, considerando que essa faixa etária raramente é testada, o que compromete as pesquisas nessa faixa etária, pois por se tratar de núme ros tão baixos, pouca atenção científica e governamental é dada para o vírus nos estágios iniciais da doença. Em 31/01/20, havia 1.179 pessoas infectadas na China; destes, $(0,6 \%)$ com idade entre 1,5 e 18 anos. Assim sendo, a atenção foi voltada para a ala adulta em detrimento da pediátrica (BORDET J, et al., 2020). 
Os Centros Chineses para Controle e Prevenção de Doenças (CDC) em fevereiro de 2020, relataram 44.672 casos; desses 416 pacientes (0,9\%) tinham entre 0 e 9 anos e $549(1,2 \%)$ entre 10 e 19 anos, indicando o aparecimento da doença na infância, durante a epidemia. Nos Estados Unidos, crianças menores de 18 anos correspondem a cerca de $8-10 \%$ dos casos certificados em laboratório, relatados ao CDC (ZHENG F, et al., 2020).

No que diz respeito aos cuidados das crianças, vê-se a dificuldade do isolamento destas, uma vez que necessitam do contato com os pais ou responsável, bem como para se alimentarem, entre outros tipos de cuidados. Estudos crescentes incluindo 46 artigos e 551 casos da Covid-19, mostrou que $87 \%$ das crianças foram expostas a um membro da família infectado, o que corrobora para a assertiva, bem como nas medidas protetivas de contágio pelo isolamento social. Some-se a isso que o uso de máscara não foi preconizado para menores de 3 anos, ficando mais vulneráveis ao contágio por gotículas contaminadas no ar. Uma vez que tenham entrado em contato com vírus, elas não possuem o mesmo cuidado que os adultos de evitarem levar a mão aos olhos ou a boca (DONG Y, et al., 2020).

Todo cuidado de profilaxia envolvendo a infância torna-se complicada pela incompreensão dos cuidados por essa faixa etária. Por isso, os adultos devem intensificar a medidas de higiene (como lavar as mãos frequentemente com sabão e água, trocar a roupa após retornar de um passeio a rua e evitar que seja compartilhado objetos ou mesmo brinquedos). Portanto, a transmissão da doença nos casos inf antis está intimamente ligada aos cuidados adotados pelos adultos (SWANN OV, et al., 2020).

Nos Estados Unidos, de 149.760 casos (02/12 a 04/02/2020), 1,7\% <18 anos; 5,7\% das crianças foram internadas em hospitais, em comparação com $10 \%$ dos adultos. Com relação à admissão em terapia intensiva de pacientes infectados pelo SARS-CoV-2, de acordo com estudo recente realizado nos Estados Unidos, 20$40 \%$ dos adultos hospitalizados precisam estar em UTI; mas em estudos com crianças, esse número é de quase $0,6-5 \%$ (ALONSO IAF, 2021).

A Covid-19 af etou 2.143 crianças, das quais 113 (5,6\%) apresentaram insuficiência respiratória ou hipóxia, enquanto $13(0,6 \%)$ evoluíram para Consenso de Síndrome do Desconforto Respiratório Aguda Pediátrico (PARDS) ou A Disfunção de Múltiplos Órgãos e Sistemas (DMOS) com necessidade de UTI. Uma série nos Estados Unidos de 745 pessoas $<18$ anos (62\% hospitalização), 15 (2\%) internados na UTI, de 02/12 a 04/02/2020 (VIEIRA RSR, et al., 2020).

No entanto, divergindo do que foi apresentado acima, um estudo retrospectivo de uma única instituição terciária envolvendo 46 crianças (idades de 0 a 21 anos) em hospitais, houve uma admissão na Unidade de Terapia Intensiva Pediátrica (28\%) maior do que o relatado na literatura, e isso está fortemente associado a níveis séricos elevados de procalcitonina, pro-BNP além da alteração nas contagens de plaquetas no sangue (CHEN N, et al., 2020).

A discordância entre os estudos está vinculada ao pouco que se sabe sobre a doença, ratificando a necessidade de investimentos em pesquisas para um melhor diagnóstico e, consequentemente, melhor tratamento. A falta de conhecimento acarreta na passividade do tratamento, e, por conseguinte, na piora do cenário público de saúde (ALONSO IAF, 2021).

SIM-P é uma nova manif estação clínica da SARS-CoV-2 em crianças e adolescentes. É caracterizada por reações inflamatórias sistêmicas, como a DK e a síndro me do choque tóxico, mas os pulmões raramente são af etados. Diferentemente dos adultos em os sintomas pulmonares são um dos principais na Covid -19. A DK é uma vasculite aguda, febril, doença cardiovascular, que af eta principalmente bebês e crianças pequenas. No SIM-P, a disfunção ventricular esquerda é a manifestação mais comum em escolares e adolescentes (CAMPOS LR, 2020).

Possui um ligeiro efeito nos vasos sanguíneos. Disfunção ventricular esquerda é o sintoma mais comum e é mais comum em crianças em idade escolar e adolescentes. De acordo com o Ministério da Saúde, as informações de casos do SIM-P foram fornecidas por pessoas que foram hospitalizadas ou morreram com idade de 0 a 19 anos, com febre alta de 3 dias ou mais e acometimento de pelo menos 2 órg ãos (olhos, pele, 
sistema cardiovascular), alterações hematológicas e/ou fortes manifestações intestinais (DAVIES P, et al., 2020).

Segundo Alonso IAF (2021), a descrição dos casos de SIM-P afirma: pessoas hospitalizadas ou que morrem entre zero e 19 anos; a presença de febre alta por três dias ou mais; envolvimento de, pelo menos, dois órgãos (olhos, pele, sistema cardiovascular, entre outros); evidência laboratorial de aumento dos sintomas inflamatórios; além de eliminar quaisquer causas de origem infecciosa; e prova de infecção por SARS-CoV-2 (laboratório ou história de contato com casos).

Alguns fatores importantes também são mencionados, como: sintomas intestinais ocorrem em mais de $70 \%$ dos casos, e dor abdominal é o mais comum. Dos pacientes acometidos $68 \%$ necessitam de cuidados de UTI; insuficiência cardíaca é o mais comum; aproximadamente $77 \%$ dos casos requerem suporte hemodinâmico, necessitando de drogas vasoativas e/ou reanimação com fluidos; $94 \%$ indicaram evidência aumentada de atividade inflamatória; radiografias pulmonares mostraram que $41 \%$ dos pacientes tiveram alterações; apenas 1,5\% morreram, indicando a importância da terapia antirretroviral e seu impacto nos resultados. Os pacientes relatados expressaram uma série de sintomas e / ou alterações laboratoriais e de imagem que confirmaram o diagnóstico de SIM-P (CAMPOS LR, 2020).

Atualmente, muitos estudos estão tentando encontrar a base para a resposta imune do SARS-CoV-2, com o objetivo de tornar o tratamento eficaz para a doença, o que tem sido dificultado pela rápida transmissão da doença e seu agravamento nos pacientes. Um estudo com 48 pacientes mostrou que adultos e crianças têm respostas imunológicas diferentes ao Covid-19. Em pacientes pediátricos, a resposta imune é causada principalmente poranticorpos $\lg G$, enquanto em adultos, todos os tipos de anticorpos podem causar respostas imunes (VIEIRA RSR, et al., 2020).

Em comparação com as crianças com SIM-P, a carga viral de pacientes idosos é significativamente menor uma vez que os pacientes pediátricos apresentam uma resposta autoimune aos anticorpos lgG, acredita-se que a SIM-P pode ser detectada durante a inf ecção por SARS-CoV-2. Um estudo comparando os resultados cardíacos de pacientes com DK e SIMP, mostrou que os pacientes com SIM-P eram geralmente mais velhos do que os pacientes com DK, com idade mínima de SIM-P de 11,4 anos, enquanto a média de DK encontrada é de 3,1 anos (GODFRED-CATO $S$, et al., 2020).

O envolvimento coronário foi mais comum em pacientes com DK, em comparação com pacientes com SIM-P. Na ecocardiografia, os pacientes com SIM-P apresentam insuficiência ventricular grave. O estudo concluiu que a manifestação mais comum no período crítico do SIM-P é o choque cardíaco, o que não é comum em pacientes com DK (HILLESHEIM D, et al., 2020).

Vale ressaltar que alguns fatores como a falta de intervenção governamental com políticas públicas que visem proporcionar à população, condições para se protegerem, tais como: uso de máscaras e produtos para desinfecção das mãos e orientações pertinentes quanto aos cuidados no distanciamento social, são impreteríveis no combate a disseminação da doença. A não informação sobre esses tipos de cuidados, faz com que a população subestime e não compreenda os riscos envolvidos nessa pandemia (GODFRED -CATO S, et al., 2020).

O governo, responsável pela saúde pública, tem o dever de mitigar os danos e providenciar as medidas necessárias, como a vacina. Desigualdades sociais e longo prazo na tomada de decisões em saúde social, como moradia, instabilidade econômica, segurança e condições de trabalho de cuidadores e familiares de pacientes, colocam as pessoas em maior risco de Covid-19 e doenças em formas mais graves (VIEIRA RSR, et al., 2020).

Algumas condições mostram choque causado por insuficiência cardíaca, com ou sem modificação da artéria coronária. Sintomas respiratórios podem estar presentes, geralmente causados por choque concomitante. $\mathrm{O}$ exame clínico completo, incluindo anamnese, exame físico, vínculo epidemiológico e casos suspeitos ou confirmados de Covid-19 nas últimas 2-4 semanas, além da solicitação de exames complementares para avaliação do processo inflamatório e envolvimento do órgão, é fundamental para suspeitas diagnóstico e tratamento (CHEN N, et al., 2020). 
Por causa das semelhanças clínicas entre as síndromes citadas, e que ambas podem ser causadas pelo coronavírus, há necessidade de preparar os profissionais de saúde para diagnosticarem e intervirem precocemente em ambos os casos, encaminhando os pacientes para unidades que já tenham experiência nesse tipo de tratamento (DONG Y, et al., 2020).

As políticas públicas são de grande valia como foi demonstrado, uma vez que impacta diretamente a vida das pessoas, reforçando a conscientização da população quanto ao isolamento social e o uso de equipamento de proteção individual, como máscaras descartáveis em locais públicos. Os cuidados preventivos devem ser estendidos às crianças pelas complicações que podem ser geradas no caso de infecção por coronavírus (VIEIRA RSR, et al., 2020).

\section{CONSIDERAÇÕES FINAIS}

Acredita-se que haja uma inflamação na infância que pode estar associada à infecção por SARS -CoV-2, semelhante à da doença de Kawasaki. Pode-se enfatizar também a necessidade de acompanhamento de longo prazo dos pacientes infectados com SIM-P para avaliar os potenciais ef eitos colaterais associados à nova infecção por coronavírus. A SIM-P compartilha características clínicas e laboratoriais da DK (crônica ou incompleta). A hipótese de uma possível interação com a infecção pelo SARS-CoV-2 foi levantada. Essas crianças desenvolvem febre crônica, erupção cutânea e sintomas abdominais proeminentes, conjuntivite, linfadenopatia, irritabilidade e dor de cabeça. Esta revisão trouxe a contribuição relativa a atualização da literatura da área de Medicina acerca da temática apresentada.

\section{REFERÊNCIAS}

1. ALONSO IAF, et al. Doenças cardiovasculares em crianças com síndrome inflamatória multissistêmica decorrente de infecção por covid-19: uma revisão integrativa. Brazilian Journal of Development, 2021;7(7): 66327-66341.

2. BORDET J, et al. Paediatric multisystem inflammatory syndrome associated with COVID-19:filling the gap between myocarditis and Kawasaki?. European journal of pediatrics, 2020;180(3):877-884.

3. CAMPOS LR, et al. Síndrome inflamatória multissistêmica pediátrica (MIS-C) temporariamente associada ao SARSCoV-2. Resid Pediatr. ,2020; 10(2): 1-6.

4. CATTALINI M, et al. Defining Kawasaki disease and pediatric inflammatory multisystem syndrometemporally associated to SARS-CoV-2 infection during SARS-CoV-2 epidemic in Italy: results from a national, multicenter survey. Pediatr Rheumatol Online J., 2021; 19(1):29.

5. CHEN N, et al. Epidemiological and clinical characteristics of 99 cases of 2019 novel coronavirus pneumonia in Wuhan, China: a descriptive study. Lancet, 2020;395(10223):507-513.

6. DAVIES P, et al. Intensive care admissions of children with paediatric inflammatory multisystem symdrome tem porally associated with SARS-CoV-2 (PIMS-TS) in the UK: a multicentre observational study. Lancet Child Adolesc Health, 2020;20: 30215-30217.

7. DONG Y, et al. Epidemiology of COVID-19 among children in china. Pediatrics, 2020; 145(6): e20200702.

8. FELDSTEIN LR, et al. Multisystem inflammatory Syndrome in U.S. Children and adolescentes. N Engl J Med, 2020; 383(4):334-346.

9. GODFRED-CATO S, et al. COVID-19- Associated Multisystem Inflammatory Syndrome in Children - United States, MarchJuly 2020. Morbidity and Mortality Weekly Report (MMWR), 2020; 69(32):1074-1080.

10. HAN Q, et al. Coronavirus 2019-nCoV: A brief perspective from the front line. J Infect., 2020;80(4): 373-377.

11. HILLESHEIM D, et al. Síndrome respiratória aguda grave por COVID-19 em crianças e adolescentes no Brasil:perfil dos óbitos e letalidade hospitalar até a 38a Semana Epidemiológica de 2020. Epidemiol. Serv. Saúde, 2020; 29(5): e2020644.

12. HOANG A. et al. COVID-19 in 7780 pediatric patients: a systematic review. Eclinical Medicine, 2020;24:1-19.

13. KABEERDOSS J, et al. Severe COVID19, multisystem inflammatory syndrome in children, and Kawasaki disease: immunological mechanisms, clinical manifestations and management. Rheumatology International, 2020;41:19.

14. SARZAEIM M, REZAEI N. Kawasaki Disease and Multisystem Inflammatory Syndrome in Children with COVID-19. SN Comprehensive Clinical Medicine, 2020;2:2096.

15. SWANN OV, et al. Clinical characteristics of children and young people admitted to hospital with covid -19 in United Kingdom :prospective multicentre observational cohort study. BMJ, 2020;370:3249.

16. VIEIRA RSR, et al. Clinical Characteristics in Children and Adolescents with SARS-CoV-2 Infection: Experience in a highly complex Public Hospital in the city of Sao Paulo. MedRXiV, 2020.

17. WANG Y, et al. Children hospitalized with Severe COVID-19 in Wuhan. Pediatr Infect Dis J., 2020; 39(7): 91-94.

18. ZHENG F, et al. Clinical Characteristics of Children with Coronavirus Disease 2019 in Hubei, China. Curr Med Sci., 2020;40(2): 275-280. 